\title{
Einstein, Entropy and Anomalies
}

\author{
Daniel Sirtes* and Eric Oberheim ${ }^{\dagger}$ \\ *Science Studies Program, University of Basel, Missionsstr. 21, 4003 Basel, Switzerland \\ ${ }^{\dagger}$ Institut für Philosophie, Humboldt-Universität zu Berlin, Unter den Linden 6, 10099 Berlin, Germany
}

\begin{abstract}
This paper strengthens and defends the pluralistic implications of Einstein's successful, quantitative predictions of Brownian motion for a philosophical dispute about the nature of scientific advance that began between two prominent philosophers of science in the second half of the twentieth century (Thomas Kuhn and Paul Feyerabend). Kuhn promoted a monistic phase-model of scientific advance, according to which a paradigm driven 'normal science' gives rise to its own anomalies, which then lead to a crisis and eventually a scientific revolution. Feyerabend stressed the importance of pluralism for scientific progress. He rejected Kuhn's model arguing that it fails to recognize the role that alternative theories can play in identifying exactly which phenomena are anomalous in the first place. On Feyerabend's account, Einstein's predictions allow for a crucial experiment between two incommensurable theories, and are an example of an anomaly that could refute the reigning paradigm only after the development of a competitor. Using Kuhn's specification of a disciplinary matrix to illustrate the incommensurability between the two paradigms, we examine the different research strategies available in this peculiar case. On the basis of our reconstruction, we conclude by rebutting some critics of Feyerabend's argument.
\end{abstract}

Keywords: Einstein, Kuhn, Feyerabend, incommensurability, pluralism, Brownian Motion, Thermodynamics, Philosophy.

PACS: 01.70.+w, 01.65.+g, 05.70.-a, 05.40.Jc

\section{DOGMATISM VERSUS PLURALISM IN THE PURSUIT OF PROGRESS}

Albert Einstein undoubtedly influenced the philosophy of science in many ways. This paper assesses the significance of Einstein's prediction of Brownian motion (henceforth BM), and Perrin's subsequent experimental confirmation, for a dispute about the functional roles of dogmatism and pluralism in promoting scientific progress. This dispute began in the early 1960s between two of the twentieth centuries most prominent philosophers of science: Thomas Kuhn and Paul Feyerabend, and it continues even today.

Kuhn, in his well-known book, The Structure of Scientific Revolutions (1962), suggests that the natural sciences differ from other fields of inquiry in the kind of progress that they achieve. His historical investigations led him to develop a phase-model of scientific advance that distinguishes two kinds of progress: normal and revolutionary. Normal science is continuous and cumulative. It progresses by solving puzzles delineated by a 'paradigm', or group of researchers sharing basic assumptions about the fundamentals of their field. Normal puzzle-solving research generates anomalies - puzzles that resist solution. If the anomalies become significant enough, they can cause a crisis state, or extra-ordinary phase of research. Basic principles and entrenched theoretical assumptions are now open to possible revision. If new basic principles allow for the dissolution of the anomaly, and can replace the older principles, then a scientific revolution has occurred. This is followed by the onset of a new puzzle-solving phase based on those new principles $\left[^{1}\right]$.

Kuhn's account of scientific advance assigns positive functional roles to dogma. Dogmatism keeps the communities focused, so that the potential of a successful paradigm can be exhausted, and it identifies the anomalies that lead to revolutionary improvements. This positive role should not be surprising. After all, on Kuhn's account, paradigms drive the puzzle-solving activities of 'normal' scientific research. Kuhn's account of scientific advance has both a descriptive and a normative element: As a historian, he claims that scientists do in fact stick to the accepted paradigm. As philosopher, he claims that this is as it should be. Otherwise science would not make the special kind of progress that it does. By insisting that faithful adherence to a single accepted paradigm was essential to the progressive nature of the natural sciences, Kuhn defended a dogmatic element in his mono-paradigmatic

CP861, Albert Einstein Century International Conference, edited by J.-M. Alimi and A. Füzfa

(C) 2006 American Institute of Physics 978-0-7354-0359-8/06/\$23.00 
account of scientific method, according to which theory proliferation is only fruitful in crisis states. This functional role of dogma in Kuhn's mono-paradigmatic account denies a role for alternatives in promoting progress in normal science by insisting that the anomalies responsible for revolutions are generated by the paradigm's normal puzzlesolving activity: "anomaly appears only against the background provided by the paradigm" $\left[{ }^{1}, 65\right]$.

Kuhn and Feyerabend make many controversial claims about scientific revolutions, most notably that they produce incommensurability. By this they mean that the paradigms offer mutually exclusive theoretical perspectives. For example, they argue that one can understand matter and motion according to the paradigm provided by Newton's mechanics, or one can understand it from the relativistic paradigm provided by Einstein's dynamics - but one cannot use the concepts of both of these two paradigms at the same time. Similarly, one can either understand the universe according to the atomist paradigm, or according to the energeticist paradigm. Kuhn and Feyerabend build on insights made by Kant and Duhem, arguing that such deep-seated theoretical commitments determine how humans experience the world. Kuhn emphasized that a consequence of incommensurability is that when the proponents of paradigms enter into debates about paradigm choice, their arguments are necessarily circular. Each group of researchers uses its own paradigm to argue in that paradigm's defense, so that the arguments appear circular from the opposing theoretical perspective. Such circular arguments cannot be made compelling for someone who refuses to step into the circle. The premises and values shared by the opposing paradigms are insufficient for that $\left[{ }^{1}, 94,261\right]$.

Kuhn and Feyerabend also agree that there is no neutral algorithm for theory-choice. When it comes to adopting paradigms in extra-ordinary phases of scientific advance, there is no systematic decision procedure, which properly applied, necessarily leads each rational individual in the research community to the same conclusions. Kuhn stresses that it is the community of specialists, rather than its individual members, that make the effective decision. To understand why science develops as it does, unraveling the details of biography and personality that leads each individual to a particular choice is unnecessary. The key to understanding scientific advance lies in the manner in which a particular set of shared values interacts with the particular experiences shared by a community of specialists ensuring that most members of the group will ultimately find one set of arguments decisive rather than another $\left[{ }^{1}\right.$, 200]. Feyerabend mainly struggles with a related issue. Convinced that theoretical perspectives determine how researchers experience the world, he set out to investigate how it is still possible for empirical evidence to force a revolution between all-pervasive, deeply entrenched theories: How can there be crucial experiments between incommensurable theoretical perspectives? $\left.{ }^{7}\right]$

While there are many points of agreement between Kuhn and Feyerabend's views, there was a deep disagreement about the function of dogma in promoting progress. Kuhn defends a dogmatic aspect of normal scientific advance on historical grounds. Feyerabend criticizes Kuhn's defense of the functional role of dogmatism. Having been heavily influenced by Popper's critical rationalism (we propose, nature disposes), he rejected all forms of dogmatism in the natural sciences. According to Feyerabend (and Popper), what distinguishes the natural sciences form other disciplines is exactly its rejection of any and all forms of dogmatism. On this popular view, science is essentially critical. Theories are developed and then tested against the evidence, and every assumption is and should be open to test in the process. The more thoroughly a theory is tested, the more empirical content it has. If a theory is falsified, then new theories should be developed to take their place.

Feyerabend developed an argument for pluralism in the natural sciences, which he used to criticize Kuhn's defense of the functional role of dogmatism. He argues that in the history of science, there was at least one case in which the anomaly that led to a scientific revolution could only undermine the existing paradigm only after an alternative theoretical perspective had been developed. BM could only be shown to refute classical thermodynamics only after statistical thermodynamics was developed - which Einstein need to make the novel prediction that served as a crucial experiment between the two incommensurable theories.

In this discussion, we develop Feyerabend's argument for pluralism against Kuhn's defense of dogmatism. We argue that Einstein's prediction and Perrin's subsequent experimental confirmation of the statistical behavior of the Brownian particles is an example of a crucial experiment between two incommensurable paradigms; and the fact that statistical mechanics was necessary to refute classical thermodynamics undermines Kuhn's mono-paradigmatic account of scientific advance. The existing evidence prior to Perrin's confirmation was inconclusive. Although Gouy's concomitant variation experiments had convinced some scientists that BM posed a serious threat to classical thermodynamics, the empirical evidence remained indecisive. Just as Kuhn's methodology predicts, they convinced only members of the atomist paradigm. Energeticists continued to deny that BM refuted their paradigm right up until Perrin experimentally confirmed Einstein's statistical predictions.

We begin by very briefly presenting a case for the claim that Perrin's experimental confirmation of Einstein's novel prediction was in fact a crucial experiment in the historical development of thermodynamics (section 2). Then we reconstruct and corroborate Feyerabend's argument that BM could have been recognized as a refutation of 
classical thermodynamics only after the development of an alternative theoretical perspective (section 3). With Kuhn's more detailed conception of incommensurability as mutually exclusive disciplinary matrices, in section 4 we illustrate the depth of the divide between atomism and energetics. This allows us to understand the relations between $\mathrm{BM}$ and the two paradigms, and the various research strategies available to them. In section 5 we use these insights to defend and strengthen Feyerabend's argument against criticism. Section 6 draws together some conclusions.

\section{EINSTEIN'S PREDICTION AND PERRIN'S CONFIRMATION}

Einstein considered his predictions of BM to be a potential crucial experiment between classical thermodynamics and the molecular-kinetic theory of heat (statistical thermodynamics) as applied to small systems even before the experiments were actually performed. His 1905 paper on BM began, "If the movement discussed here can actually be observed ... then classical thermodynamics can no longer be looked upon as applicable with precision to bodies even of dimensions distinguishable in a microscope; ... On the other hand, had the prediction of this movement proved to be incorrect, a weighty argument would be provided against the molecular-kinetic conception of heat" ${ }^{2}$, 549]. From 1908 to 1911, Perrin experimentally confirmed those predictions. From 1905 to 1913 [ $\left.{ }^{3}\right]$, members of both the atomist and energeticist paradigms gradually accepted the statistical thermodynamic explanation of BM. In 1912 v. Smoluchowski drew all the evidence together and concluded, "So, how does it stand with the second law? In the usual version of Clausius or Thomson it is surely not right, as we can see, that bodies can set oneself in motion as a result of their content of heat that they can perform work against the force of gravitation or magnetic force, so that automatically density and concentration difference appear etc., and the magnitude of these fluctuations is after all, like the magnitude of molecular velocities, totally unlimited"'[4, 1078].

Discussing that paper, Ornstein first suggested revising classical thermodynamics by restricting it to large systems where statistical fluctuations rarely have significant effects. Such revisions can be found in contemporary textbooks. Consequently, even the most orthodox adherents of classical thermodynamics (e.g. Ostwald) were convinced that the theory had only a limited validity, and that its energeticist metaphysics must be abandoned. Einstein's novel predictions (1905) had set the stage for Perrin's experimental confirmation (1908-1911) that acted as a crucial experiment, completing a revolution in the development of thermodynamics.

\section{FEYERABEND’S ARGUMENT FOR PLURALISM}

Feyerabend often argued that because established theories can avoid refuting evidence by ad hoc means, alternative theoretical perspectives are needed to force established theories to face up to potential anomalies, which otherwise are put aside. This is a lesson he learned in the 1950s through collaboration with v. Ehrenhaft regarding his experiments on magnetic monopoles. Targeting Kuhn's popular mono-paradigmatic account, Feyerabend directed his argument for the proliferation of theories against Kuhn's defense of the functional role of dogmatism, claiming that the proliferation of theoretical alternatives provides better opportunities to test theories than the simple puzzle-solving activities of normal science; and consequently, that pluralism is preferable to theoretical monism $\left(^{5}\right)$. Moreover, Feyerabend argued that the refuting character of the empirical evidence could be proven on the basis of measurements that became possible only after the development of an incommensurable competitor (the kinetic theory), and Einstein's use of it to make calculations of the statistical properties of BM. (Feyerabend drew the example from Bohm $\left.\left.{ }^{6}\right]\right)$. Perrin's confirmation of Einstein's novel prediction of the statistical behavior of the Brownian particle became Feyerabend's paradigm case of a crucial experiment between incommensurable theories $\left[{ }^{7}\right]$.

Feyerabend's argument turns on the claim that an investigation of the observational consequences of classical thermodynamics by itself (that is, without the use of an alternative account of heat) could not have led to the discovery that the Brownian particle is a perpetuum mobile of the second kind, refuting the second law of classical thermodynamics. According to Feyerabend, physical law excludes a direct refutation of the second law, which would require measuring the exact motion of the particle to ascertain the change in its kinetic energy, plus the energy spent on overcoming the resistance of the fluid; and it would have required precise measurements of temperature and heat transfer in the surrounding medium to establish that any loss was indeed compensated by the increase in energy of the particle and the work done against the fluid $\left[{ }^{8}, 27-29\right]$. Such measurements are beyond experimental possibilities because the measuring instruments interfere with the system. Neither the transfer of heat nor the path of the particle can be measured to the required level of precision, "The mean energy fluctuations are under certain circumstances quite considerable, they correspond e.g. for $1 \mathrm{~cm}^{3}$ of water to a displacement of the centre of mass in 
the vertical direction in the order of magnitude of a millimeter, but the experimental confirmation of it is totally hopeless given the imprecision of calorimetric measurements" [ $\left.{ }^{4}, 1077\right]$.

Feyerabend's point is not simply that the observations that refute a theory were discovered with the help of a different theory. (In the postscript, Kuhn acknowledged counter-examples such as Darwin's (unnecessary) revisions to his theory due to Kelvin's (incorrect) thermodynamic calculations of the time needed for the earth to have cooled sufficiently for the genesis of life $\left.{ }^{1}\right]$.) And his point is not that new physical measurement techniques became available, which allowed experimental physicists to avoid the measurement difficulties mentioned above. To the contrary, observations of Brownian particles were known long before the invention of the theories that used these observations to make predictions that refuted an accepted paradigm. The observations that confirmed these predictions, plotting BM, were not novel. They were available before the development of kinetic theory. Einstein described the situation, "My major aim in this was to find facts which would guarantee as much as possible the existence of atoms of definite finite size. In the midst of this I discovered that, according to atomistic theory, there would have to be a movement of suspended microscopic particles open to observation, without knowing that observations concerning the Brownian motion were already long familiar” $\left[{ }^{9}\right.$, 47]. However, without Einstein's quantitative predictions based on kinetic theory, these observations lacked significance. Thus, it was only after a competitor to the classical theory was developed that it was possible to prove experimentally that the otherwise wellknown phenomena of BM refutes classical thermodynamics. If there is even just one case in which the phenomenon that refutes an entire paradigm can be proven to refute that paradigm only after the development of an alternative, then Kuhn's account is incorrect. Historically speaking, it is not the case that the anomalies that led to revolutions were always developed out of failed puzzle-solving within that paradigm. And normatively, it is reasonable to promote the proliferation of theoretical alternatives to accepted paradigms, even if they face no significant empirical difficulties, because those alternatives may be needed even just to recognize the anomalous character of phenomena in the first place.

\section{INCOMMENSURABILITY AND EMPIRICAL ANOMALY}

We have seen that Einstein's prediction and Perrin's subsequent experimental confirmation of BM was considered to be a crucial experiment. And we have reconstructed and substantiated Feyerabend's argument for pluralism. It remains to be shown that the two paradigms were incommensurable in Kuhn's sense, and then to investigate the status of the empirical evidence prior to Perrin's confirmation.

Both Kuhn and Feyerabend agreed that classical thermodynamics is incommensurable with statistical thermodynamics. They both recognized that the concepts used in their respective thermodynamic laws are mutually exclusive $\left.{ }^{1,7}\right]$. But Kuhn and Feyerabend had significantly different notions of incommensurability. For example, Feyerabend usually restricted the notion of incommensurability to just three episodes of major theoretical transitions: from impetus theory to Newtonian mechanics, classical thermodynamics to kinetic theory, and Newtonian mechanics to relativity theory $\left[^{7}\right]$. These episodes are special, according to Feyerabend, because they involve universal theories - or theories that have no restrictions on their domain of application. Feyerabend believed that such deep-seated, all-pervasive theoretical perspectives generate worldviews that determine how researchers experience the world. For this reason, he was interested in how it is still possible for such all-pervasive perspectives to be empirically refuted. Moreover, Feyerabend applied the term incommensurable to the relationship between sets of concepts used to express the respective laws of the competing theories, claiming for example, that the concept of temperature in the laws of classical thermodynamics is incommensurable with the concept of temperature in the laws of statistical thermodynamics.

By contrast, Kuhn's saw incommensurability whenever there was a significant conceptual change, regardless as to the scope of the theories in question, and he did not apply the notion to single pairs of concepts, but to entire paradigms - or the research communities' shared theoretical commitments. For example, incommensurability arose between the proponents of phlogiston theory and oxidation theory of combustion or between Ptolemaic and Copernican views of the solar system. On Kuhn's holistic approach, the members of a paradigm share a group of inter-related theoretical commitments called 'the disciplinary matrix', which breaks down into four basic components: symbolic generalizations, models, values and exemplary problem solutions. On Kuhn's account of scientific knowledge, these elements form $a$ unity in that understanding thermodynamics necessarily requires a mastery of the interplay between all four elements. Incommensurability, then, holds between competing groups of scientists using the shared elements of their disciplinary matrix. A look at the stark contrast between the different symbolic generalizations, models, values and exemplars serves to illustrate this holism, and the incommensurability that it generates. 
The symbolic generalizations take the form of the respective theoretical laws shared by the paradigm. In the energetists paradigm, these include the two laws of phenomenological thermodynamics: $\Delta \mathrm{U}=\Delta \mathrm{Q}+\Delta \mathrm{W}$ and $\Delta \mathrm{S} \geq 0$, as well as entropy $\Delta S=\Delta Q / T$. By contrast, the atomist paradigm endorse the statistical thermodynamics law of equipartiton of energy $\mathrm{E}=3 / 2 k \mathrm{~T}$, entropy $\Delta \mathrm{S}=k \ln \left(\mathrm{W}\left(\mathrm{N}_{1}, \mathrm{~V}_{1}, \mathrm{U}_{1}\right) / \mathrm{W}\left(\mathrm{N}_{2}, \mathrm{~V}_{2}, \mathrm{U}_{2}\right)\right.$, and the state equation of ideal gases $\mathrm{pV}=\mathrm{NkT}$. Each paradigm is forced to choose between one set of equations. There is no consensus with respect to the logical form of the laws governing thermodynamic phenomena.

The difference in the models of the two theories is evident, in the very names of the two paradigms. Whereas the atomists supported an atomistic model of matter, the energeticists believed in the continuity of matter. Atomists conceive of the universe as containing finite particles of matter, where as energetists conceive of the universe as continuous and in permanent vibratory movement, with intersecting waves propagating in different directions, whose intersections result in limited portions of matter [see e.g. ${ }^{10}$ and ${ }^{11}$, 19f].

The differences in the values (or philosophies) of the two paradigms can be illustrated by considering the views of the two main antagonists, Boltzmann and Ostwald. In 1890 at a lecture at the Graz University, Boltzmann declared, "In my opinion, the task of a theory consists in the construction of an image of the outer world existing only inside of us, that is serving us as a lodestar in all our thoughts and experiments ... And the more abstract it is, the more powerful theoretical research will be" ${ }^{12}$, 77f, our translation]. Ostwald, on the other hand, took the fact that the terms in Gibbs' thermodynamic equations represent energy in different forms very seriously. He accused the atomists of generating pseudo-problems: hypotheses for which it was impossible to demonstrate experimentally any real content. He had been heavily influenced by Comte's positivistic philosophy, claiming "It's not our business to see the world more or less deformed in a curved mirror; we have to see it directly, as much as our intellectual abilities allow us” $\left[{ }^{13}, 956\right.$, our translation]. Boltzmann belonged to a paradigm that valued building abstract mechanistic models, whereas Ostwald thought that these kinds of models were futile and that (differential) equations and the experimental confirmation of their consequences were all a scientist could and should hope for.

Statistical and phenomenological thermodynamics also have different exemplary problem solutions. Energeticist research was guided by observations of the critical point and liquefaction of gases, along with studies of chemical equilibrium and the application of Gibbs' phase rule, Le Chatelier's principle, and Duhem's thermodynamic potential [For a discussion, see ${ }^{11}$, 13f]. Atomists, on the other hand, were guided by the problem solutions in pure chemistry provided by Dalton and Lavoisier, and later by Maxwell and Boltzmann's kinetic gas theory, which explained fundamental properties of gases such as pressure and diffusion.

Each of the elements of the disciplinary matrices of the two paradigms were clearly different and mutually exclusive, which illustrates what Kuhn meant by calling the two paradigms incommensurable, and what he meant with his infamous claim that the proponents of competing paradigms work in different worlds $\left.{ }^{1}, 150\right]$. Because the members of competing research communities share different values and understand thermodynamic phenomena according to different laws, models and exemplars, they experience the phenomena differently.

In addition to sharing mutually exclusive theoretical commitments, there are three further criteria for Kuhn's notion of incommensurability. First, the two paradigms must target roughly the same domain. Second, there must be a shift in the conceptual structure shared by the research community (what Kuhn called 'the structure of the lexicon') significant enough to make exact translation of the concepts used by the competing paradigm impossible. And third, there must be a difference in what counts as a legitimate puzzle. We will illustrate each of these criteria in turn.

In contemporary textbooks of phenomenological thermodynamics, the theory is either immediately applied to thermodynamic systems without qualifications [e.g. ${ }^{14}$ ], or it is noted in the introduction that "the system ... must be big enough, that the statistical fluctuations of the system's parameter (energy, number of molecules, etc.) will remain negligibly small" [ ${ }^{15}, 2$, our translation]. This was not the case at the turn of the century. It resulted from Perrin's experimental confirmation of Einstein's novel prediction of BM. Prior to these revisions, both theories were considered to be applicable to all thermodynamical phenomena, and thus have the same universal object domain.

Drawing on the idea that empirical concepts cannot be learned in isolation from each other, Kuhn argued that a single concept by itself has no meaning at all. It is only meaningful once it is connected with other concepts in a 'local holism', a conceptual net that he calls 'the structure of the lexicon' $\left[{ }^{16}, 90 f ., 159 f.\right]$. An important consequence of this feature of conceptual systems is that an exact translation is impossible. An exact translation between two theories requires systematically assigning each concept of the source language to a concept in the target language with the same meaning and extension (i.e. the objects on which a certain property or concept can be applied). However, if the lexica of the source and target languages have different structures, and the difference of structure is such that the respective concepts do not have the same extension, then the necessary condition cannot be fulfilled ${ }^{16}$, 256]. 
In the two respective theories of thermodynamics, the concepts 'temperature', 'energy', 'heat' and 'entropy' constitute a local holism. The equations above give the impression that the concepts of the respective theories are 'knitted' together completely differently. The meanings and the extensions of the concepts are not identical. Classical thermodynamics distinguishes between the mechanical motion of a particle and the heat of a liquid $\left[{ }^{8}, 29\right]$. This is not the case in statistical thermodynamics. A single molecule that bounces around in a container can have a temperature according to the law of equipartition. This does not make sense for classical thermodynamics.

As we have seen, one of the functions of a paradigm is to promote normal progress by guiding the identification of new research problems. According to Kuhn, three criteria must be fulfilled for the identification of new research problems. First, the community needs to share a suitable system of concepts for the satisfactory articulation of the unsolved problem. Second, they need to share a theoretical background against which the issue can be seen as a specific problem. And third, the paradigm must deliver the expectation that the problem should be solvable on the basis of the shared concepts and theoretical background of the research community $\left[{ }^{16}, 162\right]$.

The situation for BM is quit peculiar. Although BM had been accepted as a physical phenomenon in 1928 [ $\left.{ }^{17}\right]$ (it could not be distinguished from the movements of Leeuwenhoek's 'animalcules' until after the technical advance of the achromatic lens), it was still unclear as to what kind of physical phenomenon it was. This uncertainty allowed classical thermodynamicists several strategies. For example, they could treat it as a candidate for a legitimate puzzle, and then try to solve it. Sigmund Exner, who suggested that it could be caused by small currents in a medium emanating either from heat or light radiation, adopted this strategy. Alternatively, they could push the phenomenon out of their domain, and hope that it will be explained by different theories, or even completely dismiss it as an artificial oddity. However, once an alternative incommensurable theory of thermodynamics was developed that could treat BM as a legitimate puzzle, the situation changed dramatically. This step took place in 1888 with Gouy's experiments $\left[{ }^{18}\right]$. Once it was clear that BM could be treated as a thermodynamic phenomenon, which could potentially refute the classical formulation of the Second law, energeticists were hard-pressed to come up with a solution of their own. However, as we have seen, the direct refutation of the opponents' claim was hopeless given the imprecision of calorimetric measurements $\left[{ }^{4}\right]$. Two possible research strategies were left to the energeticist. Either they could try to show that the phenomenon is caused by a non-thermodynamic (or non-entropy-related) effect, and/or that every explanation of the competing thermodynamic theory also fails. If they could have managed the later, they'd have been saved provisionally. If they could have managed the former, they would have won. Pursuit of these two strategies is clearly discernable in the history of BM. For example, Exner (1900) and Duclaux (1908) tried to show that statistical thermodynamics renders no satisfactory explanation of BM [ $\left.{ }^{11}, 101-102\right]$, and Nye enumerates dozens of experiments and several theories (ranging from theories about small currents to surface tension and electrostatic theories) that try to explain the phenomenon as compatible with the principles of classical thermodynamics [ $\left.{ }^{11}, 21-26\right]$.

There is, of course, another possible strategy for removing the tension generated by the potential anomaly, namely, to restrict the domain of the theory, conceding that it does not apply to small systems. However, that would have undermined the functional role of dogmatism holding the paradigm together. As long as no conclusive proof had been given, the energeticist had legitimate reasons to believe that an otherwise fruitful paradigm would eventually carry the day.

\section{ATOMISM AND CONCOMITANT VARIATION}

Laymon contested Feyerabend's account of the role of Einstein's prediction and Perrin's subsequent experimental confirmation of BM claiming that it was possible to recognize that BM is anomalous to classical thermodynamics - even without the help of an alternative theory $\left[{ }^{19}\right]$. As we have seen, this would undermine Feyerabend's argument for pluralism. Laymon bases his challenge to Feyerabend's argument on Gouy's concomitant variation experiments (1888, 89 and 95).

The method of concomitant variation is one of Mill's inductive experimental methods [ ${ }^{20}$, book 3 , chapter 10]. It is used to infer causal factors by their quantitative variation together with the observation of proportional variation of the effect. Gouy's experiments, by contrast, were only excluding different kinds of factors. None of his experiments showed a concomitant variation with BM. In 1888, Gouy concluded from the lack of concomitant variation that BM occurs in closed systems, and therefore contradicts the Second law of classical thermodynamics, according to which the particle should stop. This is not the method of concomitant variation (for establishing a causal factor), but only the exclusion of factors by the method of concomitant variation.

Even so, the problem remains that in concomitant variation, one cannot be certain as to whether all possible factors have actually have been taken into consideration. That would be dogmatic as there may well be better 
accounts of the variation based on an incommensurable explanation. Couvalis, in his rebuttal to Laymon's critique of Feyerabend's argument has already pointed out that: "The method of concomitant variation could not have been used to refute the Second Law on its own because the source of the Brownian motion could well have turned out to be due to the action of an unknown energy source" $\left[{ }^{21}, 418 f\right]$. After all, Gouy reached his conclusion before the discovery of x-rays in 1895. Moreover, with the help of the Kuhnian analysis given, we can make the case for Feyerabend's argument even stronger. What Laymon and Couvalis both fail to acknowledge is that Gouy was a fervent atomist.

Laymon admits that Gouy used "Maxwell-like models". Those models are "suggested by atomic theories of random motion" $\left.{ }^{19}, 238\right]$. Laymon attempts to parry this objection with a technicality emphasizing that Gouy did not actually use the concept of an atom in his arguments. However, as we have seen in section 4, a paradigm cannot be reduced to a single concept. A paradigm consists of a disciplinary matrix of beliefs about fundamental laws, models, exemplary solutions and values. Gouy shared the beliefs of the atomistic paradigm and only because he did, it was possible for him to come to the conclusion, that BM refutes the classical conception of the Second law of thermodynamics. The alternative incommensurable paradigm of atomism was necessary for Gouy's insights. Therefore, Gouy's conclusion about the significance of BM, no matter how inconclusive it may have been at that time, must be seen as argument for the necessity of alternatives and not as a point against it.

It is no coincidence that Gouy's insight that BM is in contradiction to the Second law was first made sixty years after Brown discussed the phenomenon. Only after the formulation of kinetic theory of gases by Maxwell and Boltzmann (1866) was such a conclusion plausible. It was well known that the formulation of entropy in statistical terms clashed with the classical conception. Gouy was only the first to realize that this tension could be resolved in favor of the statistical formulation on the basis of statistical thermodynamics. His confidence in the conclusion that he had excluded all other possible explanations was motivated by his atomist beliefs, and was only (if at all) convincing to scientist who already shared the atomist view. It did not convince the energeticists, as Kuhn's claims about circular arguments in extraordinary phases predict. Furthermore, even after Gouy, the most widely accepted theory about BM was Sigmund Exner's theory that it is caused by small currents in the medium, which does not contradict the principles of classical thermodynamics. Finally, Henri Poincaré, concluded his talk at the Congress of Arts and Sciences in St. Louis in 1904, presenting Boltzmann's ideas and the results of the experiments of Gouy on the exclusion of concomitant variation with these words: "I hasten to say we are not yet there, and as yet nothing proves that the principles [of classical thermodynamics] will not come forth from the combat victorious and intact" (Poincaré, 1904)

\section{CONCLUSIONS}

We have shown that Einstein's successful prediction of the Brownian motion was a crucial experiment between two incommensurable paradigms. With the help of Kuhn's insights into the holistic nature of scientific research we strengthened and defended Feyerabend's argument that the Brownian motion could have been recognized as a refutation of classical thermodynamics only on the basis of an alternative paradigm. We have shown that Gouy's entrenchment in the atomistic paradigm was a necessary condition for his conviction that his exclusion of concomitant variation was sufficient to establish that Brownian motion is relevant to the Second law of classical thermodynamics. However, Gouy's experiments did force the energeticist to recognize the potential refuting character of the phenomenon, thus importing a research problem into an incommensurable paradigm. Therefore, Gouy's paper on Brownian motion supports Feyerabend's argument instead of, as a critic had suggested, undermining it.

Finally, Kuhn's positive evaluation of dogma fails in the face of Feyerabend's argument for pluralism. This in principle argument has immediate consequences for science policy, as it implies that we cannot know how much we may be missing out on without the development of alternative theories. Even if our best theories face no serious empirical anomalies and are taken to be extremely fruitful, it is impossible to judge their true merits without the contrast only alternatives can provide. Proliferate for progress.

\section{ACKNOWLEDGMENTS}

We would like to thank Paul Hoyningen-Huene for helpful comments on a draft of this paper. 


\section{REFERENCES}

T. S. Kuhn, The Structure of Scientific Revolutions, 2nd ed. ed. (University of Chicago Press, Chicago, 1970).

A. Einstein, Annalen der Physik 17, 549 (1905).

J. Perrin, Les Atomes. (Aclan, Paris, 1913).

M. v. Smoluchowski, Physikalische Zeitschrift XIII, 1069 (1912).

P. K. Feyerabend, in Criticism and the Growth of Knowledge, edited by I. Lakatos and A. Musgrave (Cambridge

University Press, Cambridge, 1970), pp. 197.

D. Bohm, Causality and Chance in Modern Physics. (Routledge, London, 1957).

P. K. Feyerabend, in Scientific Explanation, Space, and Time. Minnesota Studies in the Philosophy of Science, Vol. III, edited by H. Feigl and G. Maxwell (University of Minnesota Press, Minneapolis, 1962), Vol. Vol. III, pp. 28.

P. K. Feyerabend, Against Method. Outline of an Anarchistic Theory of Knowledge. (New Left Books, London, 1975).

A. Einstein, in Albert Einstein: Philosopher Scientist, edited by P. Schilpp (Cambridge University Press, London, 1949), pp. 1.

C. A. Wurtz, La Théorie Atomique. (Librairie Germer Baillière et Cie, Paris, 1879).

M. J. Nye, Molecular Reality. A Perspective on the Scientific Work of Jean Perrin. (MacDonald/American Elsevier, London/New York, 1972).

L. Boltzmann, in Populäre Schriften, edited by Ludwig Boltzmann (Leipzig, 1905), pp. 76.

W. Ostwald, Revue generale des sciences 6, 953 (1895).

D. Leuschner, Grundbegriffe der Thermodynamik. (Akademie-Verlag, Berlin, 1979).

G. Adam, P. Läuger, and G. Stark, Physikalische Chemie und Biophysik, 2nd Edition ed. (Springer, Berlin, 1988).

P. Hoyningen-Huene, Reconstructing Scientific Revolutions. The Philosophy of Science of Thomas S. Kuhn. (University of Chicago Press, Chicago, 1993).

R. Brown, Philosophical Magazine 4, 161 (1828).

L. Gouy, Journal de Physique 7, 561 (1888).

R. Laymon, Philosophy of Science 44, 225 (1977).

J. S. Mill, A System of Logic: Ratiocinative and Inductive. (Routledge, London, 1973).

S. G. Couvalis, Philosophy of Science 55, 415 (1988). 